\title{
A Swarm Intelligent Multi-path Routing for Multimedia Traffic over Mobile Ad hoc Networks
}

\author{
Saida ZIANE \\ LISSI/SCTIC Lab, \\ IUT Créteil-Vitry, Université Paris XII-Val de Marne \\ 122, Rue Paul Armangot 94400 Vitry s/Seine, France \\ Tel. +33141807374 \\ s_ziane@yahoo.fr
}

\author{
Abdelhamid MELLOUK \\ LISSI/SCTIC Lab, \\ IUT Créteil-Vitry, Université Paris XII-Val de Marne \\ 122, Rue Paul Armangot 94400 Vitry s/Seine, France \\ Tel. +33141807374 \\ mellouk@ieee.org
}

\begin{abstract}
In the last few years, the advance of multimedia applications has prompted researchers to undertake the task of routing multimedia data through Manet. This task is rather difficult due to the highly dynamic topology of mobile ad hoc networks and their limited bandwidth. Actually, different routing algorithms are proposed in order to route various kinds of sources (such as voice, video, or data) with diverse traffic characteristics and Quality of Service Requirements (QoS). These algorithms must take into account significant traffic problems such as packet losses, transmission delays, delay variations, etc, caused mainly by congestion in the networks. The prediction of these problems in real time is quite difficult, making the effectiveness of "traditional" protocols based on analytical models questionable. We propose in this paper a solution based on swarm intelligence paradigm that we find more adapted for this kind of problems.
\end{abstract}

\section{Categories and Subject Descriptors}

C.2.1 [Computer-Communication-Networks]: Network Architecture and Design - Wireless communication, Network communications, Network topology.

\section{General Terms}

Algorithms, Management, Performance, Reliability, Experimentation, Verification.

\section{Keywords}

Reinforcement learning, Adaptive Routing, Swarm intelligence, Manets, Multimedia traffic, Quality of Service.

\section{INTRODUCTION}

A mobile ad hoc network, called MANET, is an autonomous collection of mobile nodes that communicate over relatively bandwidth constrained wireless links. Since the nodes are mobile and the traffic is irregular, the network model may change rapidly and unpredictably over time. The network is decentralized, where all network activity including discovering the topology and

Permission to make digital or hard copies of all or part of this work for personal or classroom use is granted without fee provided that copies are not made or distributed for profit or commercial advantage and that copies bear this notice and the full citation on the first page. To copy otherwise, or republish, to post on servers or to redistribute to lists, requires prior specific permission and/or a fee.

Q2SWinet'05, October 13, 2005, Montreal, Quebec, Canada.

Copyright 2005 ACM 1-59593-241-0/05/0010...\$5.00. delivering messages must be executed by the nodes themselves, i.e., routing functionality will be incorporated into mobile nodes. Recently, there has been growing general interest in such networks. The advance of multimedia applications in the few last years has prompted researchers to study the problems encountered when mobile ad hoc networks carry multimedia traffic.

Mobile ad hoc networks offer connectivity in highly dynamic environment, where routers can move and signal quality change rapidly. The variation of resource availability (in particular bandwidth) is a real challenge for the delivery of high quality realtime multimedia streams.

In this case, Multi-path transmission in mobile ad hoc networks provides higher bandwidth and better guarantee on packet delivery than the traditional methods based on one shortest path. This is more suitable for multimedia applications since processing of data packets is sensitive to error and delay. Current mobile ad hoc routing protocols are classified as either reactive or proactive.

We focus in this paper on the proavtive routing and link bandwidth allocation problems. We are interested by intelligent routing which has not yet made major contributions in this kind of networks. The remainder of this paper is organized as follows: In section 2, we present briefly mobile ad hoc networks and the different algorithms proposed in the literature. In section 3 and 4, we introduce routing algorithms and we focus on those based on reinforcement learning. In section 5, we introduce swarm intelligence. In section 6 and 7, after giving an abstract of the routing protocols using swarm paradigm, we describe the solution that we propose. Finally, we finish with a conclusion.

\section{ROUTING IN MOBILE AD HOC NETWORKS}

A lot of different definitions and parameters for the concept of quality of service can be found. For ITU-T E.800 recommendation, QoS is described as "the collective effect of service performance which determines the degree of satisfaction of a user of the service". This definition is completed by the I.350 ITU-T recommendation which defines more precisely the differences between QoS and Network Performance. Relating QoS concepts in the Internet are focused on a packet-based endto-end, edge-to-edge or end-to-edge communication. QoS parameters refer to this packet transport at different layers are : availability, Bandwidth, delay, jitter and loss ratio. It's clear that the integration of these QoS parameters increases the complexity of the used algorithms. Anyway, there will be QoS relevant 
technological challenges in the emerging hybrid networks which mixed several different types of networks (wireless, broadcast, mobile, fixed, etc.).

In the literature, we can find the usage of QoS in three ways :

- Deterministic QoS consists in sufficiently resources reserved for a particular flow in order to respect the strict temporal constraints for all the packages of flow. No loss of package or going beyond of expiries is considered in this type of guarantee. This model makes it possible to provide an absolute terminal on the time according to the reserved resources.

- Probabilistic QoS consists in providing a long-term guarantee of the level of service required by a flow. For time-reality applications tolerating the loss of a few packages or the going beyond of some expiries, the temporal requirements as well as the rates of loss are evaluated on average. The probabilistic guarantee makes it possible to provide a temporal terminal with a certain probability which is given according to the conditions of load of the network.

- $\quad$ Stochastic QoS which is fixed before by a stochastic distribution.

For us, the main issues inherent in a MANET are the following :

- Conservation of power, which is essential to users of mobile wireless networks,

- Dynamic network topologies, presenting challenges in routing and link bandwidth allocation,

- Providing consistent quality of service levels subject to a changing environment.

These issues are critical for such networks because of the heterogeneous nature of nodes. Generally, nodes in a MANET, consist of laptops, personal digital assistants (PDAs), mobile phones, etc. Such equipment has different data rate requirements and limited bandwidth and power.

Due to the dynamic nature of MANETs, designing communications and networking protocols for these networks is a challenging process. One of the most important aspects of the communications process is to provide routing protocols to be used to establish and maintain multi-hop routes to allow the data changing between nodes.

A considerable amount of research has been done in this area, and several multi-hop routing protocols have been developed. Most of these protocols such as the Dynamic Source Routing protocol (DSR) [2], Ad Hoc on Demand Distance Vector protocol (AODV) [3], Temporally Ordered Routing Protocol (TORA) [4], and others establish and maintain routes on a best-effort basis.

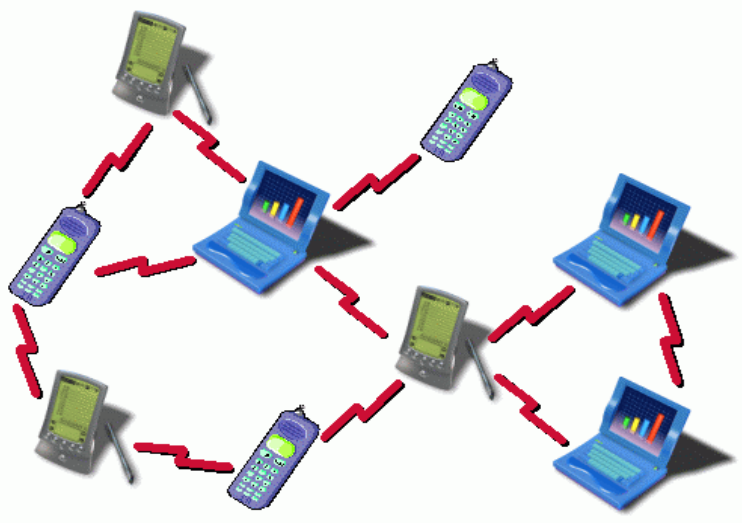

Figure 1: Mobile ad hoc network

While this might be sufficient for a certain class of MANET applications, it is not suitable for the support of more demanding applications such as multimedia audio and video. Such applications require the network to provide guarantees on the Quality of Service (QoS).

The task of finding suitable methods to carry multimedia packets through mobile ad hoc networks is rather difficult but not impossible. In [5],[6], and [7], a method based on a set of multi paths to carry split video streams has been proposed.

In MANETs, nodes are responsible to establish automatically routing among themselves. So, each node in the network is considered as a router which forwards packets to other nodes. A natural method to provide routing in ad hoc networks is to run a conventional routing protocol between them. Unfortunately, there are some problems with this approach:

- Transmission between two hosts over a wireless network does not necessarily work equally well in both directions,

- The redundant paths in the wireless networks unnecessarily increases the size of routing updates that must be sent over the network, and increases the CPU overhead required to process each update and to compute new routes,

- Periodically sending routing updates wastes network bandwidth and battery power,

- Finally, conventional routing protocols are not designed for the type of dynamic topology changes that may be present in ad hoc networks.

Consequently, routing protocols designed for wired networks are not suitable for ad hoc networks. Recently, a variety of routing protocols have been proposed. Traditionally all ad-hoc network protocols are classified as either proactive or reactive. Proactive protocols constantly update the network topology by exchanging route information among neighbouring nodes.

Each node maintains its own routing information readily available, so that users can immediately route packets to 
appropriate neighbours for a particular destination. The disadvantage of proactive routing protocol is that update messages are transmitted periodically even when there are minimum activities in the network. An example of a proactive routing protocol is DSDV [8].

In reactive protocols, route discovery process is only initiated when a path between a source and a destination is needed. The source node floods the network with query messages and receives routing information by accepting route reply message from either the destination node itself or other neighbouring nodes. The main disadvantage of reactive routing protocols is the excessive delay for transmission when the protocol initiates route discovery process. Examples of reactive protocol are AODV in [3], TORA in [4] and DSR in [2].

Although both reactive and proactive are not very suitable within a highly dynamic network environment, DSR was chosen for simplicity and ease of modification for multi-path transportation. According to [2]. DSR uses route request (RREQ), route reply (RREP) and route error packets to discover and maintain routes in the network. It has been suggested that route request packets have the ability to piggyback information for different network layers. However RREQ is not a suitable carrier for feedback information, but RREP on the other hand would make a better feedback carrier [7].

A new on-demand protocol named Delay Sensitive Adaptive Routing Protocol (DSARP) [9] is an on-demand routing protocol, which serves better than DSR because it uses RREP to piggyback queue length information back to the source to make multi-path decision for its packets.

Zone Routing Protocol (ZRP) [10] is another suitable protocol for the multi-path selection routing. It uses both proactive and reactive aspects of two network protocol classes. ZRP only proactively maintains the topological information of its closed neighbours to reduce the overhead of control traffic. On the other hand when it needs to find a route outside its zone, it reactively controls the flooding of query messages. The controlled flooding reduces the delay for route discovery process [7].

\section{QoS BASED ROUTING ALGORITHMS.}

The traditional ad-hoc routing protocols cannot handle the QoS in real-time traffic since their only concern is to deal with the connected and broken link of the network. Packets of data are dropped when the relaying node cannot handle the incoming traffic. In addition to packet drop, the source node has no knowledge of the current traffic condition of the network.

A lot of study has been conducted in a search for an alternative routing paradigm that would address the integration of dynamic criteria. The most popular formulation of the optimal distributed routing problem in a data network is based on a multicommodity flow optimization whereby a separable objective function is minimized with respect to the types of flow subject to multicommodity flow constraints [11], [12]. However, due their complexity, increased processing burden, a few proposed routing schemes could been accepted for the internet. We listed here some QoS based routing algorithms proposed in the literature:

- QOSPF (Quality Of Service Path First) [13] is an extension of OSPF. Combined with a protocol of reservation, this protocol of routing with quality of service makes it possible to announce to all the routers the capacity of the links to support QOS constraints.

- $\quad$ The MPLS (Multiprotocol label switching) [14], [15] technology has emerged from the need to integrate high-speed label-swapping ATM switches into IP routing networks. It introduces a connection-oriented label switching mechanism in a connectionless IP network. MPLS is a protocol which allow to assign a fixed path to the different flows toward their destination. It is based on the concept of label switching. A traffic characterization representing the required $\mathrm{QoS}$, is associated to each flow.

- $\quad$ Traffic engineering (TE) based algorithms have the goal to facilitate efficient and reliable network operations, and optimize the utilization of network resources. TE objectives can be divided into traffic oriented and resource oriented objectives. The first aims to improve the QoS characteristics of traffic stream. The second refer to the efficient use of network resources, especially bandwidth. Resource objectives should prevent congestion in one part of the network, while other part of the network, providing alternate paths are under-used. One important technique by TE is load balancing which aims to minimize maximum resource utilization. Another approaches are used in TE: Constraint-Based Routing, Explicit Routing, Using RSVP Protocol, etc. [16], [17].

- Wang-Crowcroft algorithm [18] consists of finding a bandwidth-delay-constrained path by Dijkstra's shortest path algorithm. First, all links with a bandwidth less than the requirements are eliminated so that any path in the resulting graph will satisfy the bandwidth constraint. Then, the shortest path in terms of delay is found. The path is feasible if and only if it satisfies the delay constraint.

\section{QOS BASED STATE-DEPENDENT ROUTING ALGORITHMS.}

For a network node to be able to make an optimal routing decision, according to relevant performance criteria, it requires not only up-to-date and complete knowledge of the state of the entire network but also an accurate prediction of the network dynamics during propagation of the message through the network. This, however, is impossible unless the routing algorithm is capable of adapting to network state changes in almost real time. So, it is necessary to develop a new intelligent and adaptive optimizing routing algorithm. This problem is naturally formulated as a dynamic programming problem, which, however, is too complex to be solved exactly. 
Recently, Reinforcement Learning algorithms (RL) [19], [20] have attracted the attention of many researchers in the field of dynamic routing through wired networks justified by the statistical nature of these problems and the necessity to "predict" the effects of the multiplexed traffic in the networks. Resulting routing algorithms may be better able to perform well under widely varying conditions.

A reinforcement learning algorithm is a finite-state machine that interacts with a stochastic environment, trying to learn the optimal action the environment offers through a learning process. At any iteration, the automaton chooses an action, according to a probability vector, using an output function. This function stimulates the environment, which responds with an answer (reward or penalty).

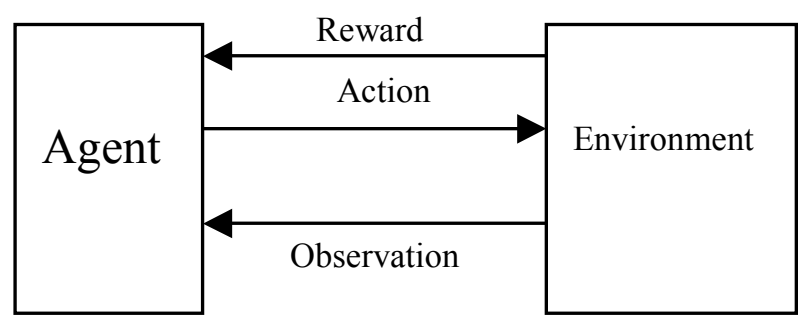

Figure2: The framework of reinforcement learning

The automaton takes into account this answer and jumps, if necessary, to a new state using a transition function [19]. There are mainly four approaches of adaptive routing using RLA.

\subsection{Q-Routing}

Q-Routing using the Q-Learning framework is proposed by Boyan and Littman [21]. In Q-Routing, each node $x$ have a routing table of values $\mathrm{Q}_{\mathrm{x}}(\mathrm{d}, \mathrm{y})$ for a neighbor $y$ and a destination $d$, of how long it takes for a packet to be delivered to node $d$ via neighbor node $y$. When the node has to make a routing decision, it simply chooses the neighbor $y$ for which $\mathrm{Q}_{\mathrm{x}}(\mathrm{d}, \mathrm{y})$ is minimum.

Value $\mathrm{Q}_{\mathrm{x}}$ is updated when message is sent from node to _ using the following equations.

$$
\begin{gathered}
T=\min _{x \in N(y)} Q_{y}(d, x) \\
Q_{x}(d, y)=\alpha\left(q_{y}+s_{y}+T\right)+(1-\alpha) Q_{x}(d, y) .
\end{gathered}
$$

Where $\mathrm{N}(\mathrm{y})$ denotes the set of neighbors of node $y, d$ denotes source node, $\mathrm{q}_{\mathrm{y}}$ denotes hold time within queue of node $y, \mathrm{~s}_{\mathrm{y}}$ denotes trip time from node $x$ to $y, \alpha$ denotes a learning rate parameter.

In this technique, each node makes its routing decision based on the local routing information, represented as a table of $Q$ values which estimate the quality of the alternative routes. These values are updated each time the node sends a packet to one of its neighbors. However, when a $\mathrm{Q}$ value is not updated for a long time, it does not necessarily reflect the current state of the network and hence a routing decision based on such an unreliable $\mathrm{Q}$ value will not be accurate. The update rule in Q-Routing does not take into account the reliability of the estimated or updated $Q$ value because it's depending on the traffic pattern, and load levels, only a few $Q$ values are current while most of the $Q$ values in the network are unreliable. For this purpose, other algorithms have been proposed like:

- CQ-Routing [22] improves over Q-Routing, by incorporating a confidence measure (C value) with each $\mathrm{Q}$ value. The $\mathrm{C}$ value denotes how closely the corresponding $\mathrm{Q}$ value represents the current state of the network. As the time, since the last update of a $\mathrm{Q}$ value, increases its $\mathrm{C}$ value decreases exponentially.

DRQ-Routing combines Q-Routing with Dual Reinforcement Learning [23], [24]. Dual reinforcement learning adds the backward exploration to the forward exploration of Q-routing, making DRQ-Routing twice as good as Q-Routing in terms of speed of adaptation (at low loads) and average packet delivery time (at high loads).

\subsection{Cognitive Packet Networks (CPNs)}

The Random Neural Network (RNN) model [25] has been the basis of theoretical efforts and applications during the last decade. It has been proven to be successful in a variety of applications when used either in a feed-forward or a fully recurrent architecture. In most problems, RNN yields strong generalization capabilities, even when training data set is relatively small compared to the actual testing data. Cognitive Packet Networks (CPNs) proposed in [25], are based on random neural networks. These are store-and-forward packet networks in which intelligence is constructed into the packets, rather than at the routers or in the high-level protocols.

CPN is then a reliable packet network infrastructure, which incorporates packet loss and delays directly into user QoS criteria and use these criteria to conduct routing. Cognitive packet networks carry three major types of packets: smart packets, dumb packets and acknowledgments (ACK).

Smart or cognitive packets route themselves, they learn to avoid link and node failures and congestion and to avoid being lost. They learn from their own observations about the network and/or from the experience of other packets. They rely minimally on routers.

When a smart packet arrives to a destination, an acknowledgment (ACK) packet is generated by the destination and the ACK heads back to the source of the smart packet along the inverse route. As it traverses successive routers, it is used to update mailboxes in the CPN routers, and when it reaches the source node it provides source routing information for dumb packets. Dumb CPN packets of a specific QoS class use successful routes, which have been selected in this manner by the smart packets of that class.

The major drawback of algorithms based on cognitive packet networks is the convergence time, which is very important when the network is heavily loaded. 


\subsection{Ant Colonies}

Inspired by dynamics of how ant colonies learn the shortest route to food source using very little state and computation, Ants routing algorithms proposed initially in [26] are described as follows. Instead of having fixed next-hop value, the routing table will have multiple next-hop choices for a destination, with each candidate associated with a possibility, which indicates the goodness of choosing this hop as the next hop in favor to form the shortest path [26]. These possibility values are initially equal and will be updated according to the ant packets pass by.

Given a specified source node and destination node, the source node will send out some kind of ant packets based on the possibility entries on its own routing table. Those ants will explore the routes in the network. They can memory the hops they have passed. When an ant packet reaches the destination node, the ant packet will return to the source node along the same route. Along the way back to the destination node, the ant packet will change the routing table for every node it passes by. The rules of updating the routing tables are: increase the possibility of the hop it comes from while decrease the possibilities of other candidates.

Compared with the real ant foragers, changing routing table is just like laying down some virtual pheromone on the way thus affects the route of the subsequent ant packets. Since the route with higher possibility is always favored, so more ants will pick up that route, and further increase its possibilities and in turn attracts more ants. With this positive feedback loop, we can expect a best path will be quickly emerged. With the changing of network load, when a new best solution comes up, we also expect that it could be identified and enforced by ant packets too. So ant routing is much more dynamic, robust and scalable.

Ants approach is immune to the sub-optimal route problem since it explores, at all times, all paths of the network. Although, the traffic generated by ant algorithms is more important than the traffic of the concurrent approaches.

\subsection{N-Best Neural Q-Routing Shortest Path}

This approach developed in [27], [28] is based on adaptive algorithm for packet routing using reinforcement learning. The Qfunction is approximated by a reinforcement learning neural network. In this approach, Neural Network ensures the prediction of parameters depending on traffic variations. Compared to the approaches based on a Q-table, the Q-value is approximated by a reinforcement learning based neural network of a fixed size, allowing the learner to incorporate various parameters such as local queue size and time of day, into its distance estimation. Indeed, a Neural Network (NN) allows the modelling of complex functions with a good precision along with a discriminating training and a taking into account of the context of the network. All these routing algorithms explore all the network environment and do not take into account loop problem in a way leading to large time of convergence algorithm.

To address this drawback and reducing computational time, [28] present the K Shortest Path Q-Routing algorithm which is based on hybrid approach combining neural networks and reducing the search space to K-Best no loop paths in terms of hops number. This approach requires each router to maintain a link state database, which is essentially a map of the network topology. When a network link changes its state (i.e., goes up or down, or its utilization is increased or decreased), the network is flooded with a link state advertisement (LSA) message. This message can be issued periodically or when the actual link state change exceeds a certain relative or absolute threshold.

\section{SWARM INTELLIGENCE}

Swarm intelligence appears in biological swarms of some social insect spices as ants. Through simple interaction of autonomous swarm members, the group behavior gives rise to complex and intelligent behavior.

Due to the use of mobile agents and stigmergy, swarm intelligence boats a number of advantages [29]:

1. Scalability: population of the agents can be adapted according to the problem size.

2. Fault tolerance: swarm intelligence do not rely on a centralized control mechanism. Therefore, the fail of a few agents does affect the reliability of the system.

3. Adaptation: agents can change according to system changes.

4. Autonomy: almost no human supervision is required.

5. parallelism: agent's operations are inherently parallel.

These properties make the swarm intelligence paradigm very attractive for networks problems as adaptive routing.

\section{SWARM INTELLIGENCE BASED ROUTING}

The basic difference between swarm based algorithms and classic routing schemes is the use of stochastic exploration for new route discovery in swarm based techniques [29]. The core of this approach is the routing table which assign probabilities to next hops and special agents that use these probabilities to choose the next hop. Agents exploring the routes are called "ants".

There are a number of swarm-based routing algorithm in the literature. The most well known one is called AntNet, it was proposed by Di Caro and Dorigo [30]. In AntNet, routing is determined by means of very complex interactions of forward and backward network exploration ants. Forward ants report network delay conditions to the backward ants. So, no node routing updates are performed by the forward ants, they only gather the useful information which will be used by backward ants to update the routing table of the nodes.

Existing swarm-based algorithms have been developed for wired networks and have several features unsuitable for mobile ad hoc networks. We have proposed in [31] a solution inspired by those proposed for wired networks and which responds to the requirements of mobile networks. 
Our algorithm is inspired by the routing algorithm proposed by subramanian in [26]. It uses three kinds of agents : regular forward ants, uniform forward ants and backward ants. Regular and uniform forward ants explore and reinforce the paths of the network proactively. They create a probability distribution at each node for its neighbors. Backward ants are used to propagate the information collected by forward ants through the network, and to adjust the routing table entries.

\subsection{Routing table}

To detect and monitor links to neighbors, a kind of special messages, called Hello, are used. Each active node periodically broadcasts a Hello message that all its neighbors receive. Hello messages are transmitted in an interval of Hello_Interval seconds. In this manner, if a node fails to receive several Hello messages from a neighbor, a link break is detected and its routing table is updated.

The routing table entries are of the form (Destination, Next-hop, Probability, AvailableBW). At first, there are no routing table entries. The creation of the first forward ant at a node for a source destination pair causes the routing table entries to be initialized with probabilities $1 / \mathrm{N}$ for each neighbor, where $\mathrm{N}$ is the number of neighbors of the node when the table is being established [32]. In a second step, these probabilities are adjusted by backward ants.

\subsection{Routing the agent packets}

Agent packets in our routing algorithm are of three kinds :

Regular forward ants: periodically, each node chooses randomly a set of destination nodes to which it sends a forward ant. Each forward ant packet contains the following fields:

- $\quad$ Source node IP address

- Destination node IP address

- Next hop IP address

- $\quad$ Stack

- Hop count

If the entry of the current destination does not exist when the forward ant is created, then a routing table entry is immediately created. The stack field contains the IP addresses of nodes traversed by forward ant packet.

Uniform forward ants: In order to keep our algorithm able to be adaptive to frequent changes in the network, we introduce, in the same manner proposed by subramanian [26], another set of ants, called uniform ants. For X\% of the time, each node sends out uniform ants instead of creating regular ants. These ants are routed by choosing the next hops with uniform probability. If the current node has $\mathrm{N}$ neighbors then the probability to choose a neighbor as the next hop in $1 / \mathrm{N}$ and it is the same for all the neighbors. These ants ensure the exploration and the reinforcement of newly discovered paths.

Backward ants: As soon as a regular or uniform forward ant reaches its destination, a backward ant is generated and the forward ant is destroyed. Backward ant inherits then the stack and the bandwidth information contained in the forward ant. The backward ants retrace the path of the forward ants by popping the stack and updating the routing tables at each intermediate node according to the following rules:

- $\quad P_{\text {id }} \leftarrow P_{\text {id }}+f(\mathrm{BW})$

- $\quad P_{\mathrm{jd}} \leftarrow P_{\mathrm{jd}}-(f(\mathrm{BW}) / \mathrm{N}-1)$

- $\quad P_{\text {id }}=1-\sum P_{\text {jd }}$.

Where $P_{\text {id }}$ is the probability of choosing the node $i$ as the next neighbor to reach the destination $d$, and $P_{\mathrm{jd}}$ is the probability of choosing another neighbor $j$ different of $i . f(\mathrm{BW})$ is the reinforcement parameter, it is a function of the available bandwidth. We assume that : $0<f(\mathrm{BW})<<1 ; f(\mathrm{BW})$ decrease proportionally with the available bandwidth. This ensure that the neighbor having the highest probability is the one with the highest available bandwidth.

\subsection{Impact of node mobility on routing tables}

When a node enter into the transmission range of a node, this can create new available routes to a destination that was reachable by a longer route or previously unreachable. The Hello messages broadcast by each node give information regarding the availability of a node as a next hop. When a node $A$ leaves the transmission range of a given node $B, A$ is then removed from the routing table of $B$. The probabilities in the routing table of the node $B$ are adjusted as follow:

- For node A as next hop, the probability is made 0 for all destinations from node $B$.

- For the other nodes, the probability distribution is normalized in a manner that the sum of the probabilities is 1 .

\subsection{Routing data packets}

At each node, we define two levels of priority. For each level we associate a queue. At first time of our proposition, we use the queue with high priority for data packets. Data packets routing is based on the probabilities at each intermediate node from the source to the destination. Arrived to a node A, the packet is routed to the node $\mathrm{B}$ if the probability of $\mathrm{B}$ is the highest of all the other neighbors.

\section{SWARM INTELLIGENT ROUTING FOR MULTIMEDIA TRAFFIC OVER MANETS}

In order to deal with problems meeting when multimedia traffic is carried over mobile ad hoc networks, and guarantee the quality of service requested by such applications, we propose in this section a solution based on our algorithm presented in the previous section. To make it more suitable for multi media traffic, our algorithm will be enhanced by multi path selection. In other words, we propose to combine swarm intelligence and multi path approaches in order to improve the QoS required by multimedia traffic carried over mobile ad hoc networks.

Naturally, a suitable multi-path selection would determine a set of shortest paths between source and destination to satisfy the realtime requirements. We propose then to exploite the information 
gathered by exploration ant packets saved in routing tables. This information consists of probabilities fields in the routing tables. So, we select a set of neighbors having higher probabilies and then instead of the use of only one neighbor having the highest probability, we have to send our multimedia packets in a multi path manner like in [33].

\section{CONCLUSION}

This paper has discribed a new QoS routing algorithm for multimedia traffic over Manets. Our scheme uses reinforcement learning techniques enhanced by multi path routing to adapt to frequent changes in the network topology and hard constraints of wireless networks.

We plan future investigations to get better our algorithm. In this optic, we will define more then two level of priority and we will implement an intelligent scheduler at each node which will dynamically schedule packets, using a swarm intelligent system.

\section{REFERENCES}

[1] S. Corson and J. Macker, "Mobile Ad hoc Networking (MANET): Routing protocol Performance Issues and Evaluation Considerations", Internet draft, draft-ietf-manetissues-01.txt.

[2] D. B. Johnson, D. A. Maltz, "Dynamic Source Routing in Ad Hoc Wireless Networks", in Mobile Computing (T. Imielinski and H. Korth, eds.), Kluwer Academic Publishers, 1996.

[3] Elizabeth M. Belding-Royer. "Report on the AODV Interop". UCSB Tech Report 2002-18, June 2002.

[4] Vincent D. Park and M. Scott Corson. "Temporally-Ordered Routing Algorithm (TORA) version 1: Functional Specification". Internet-Draft, draft-ietf-manettora -spec01. txt, August 1998.

[5] Y. Wang, S.Panwar, S.Lin and S.Mao, "Reliable Transmission of Video over Ad hoc Networks Using Automatic Repeat Request and Multi-path Transport", Proc. IEEE Fall VTC 2001, vol.2,pp615-619, Atlantic City, October 8-10, 2001.

[6] Y. Wang, S.Panwar, S.Lin and S.Mao,"Video Transport over Ad-hoc Networks Using Multiple Paths". Invited Paper, Proc. IEEE 2002 International Symposium on Circuit and Systems Scottdale, Arizona, May 26-29, 2002.

[7] M. Kwan, K. Dogancay and L. Jain, "Fair Multi-path Selection For Real-Time Video Transmission In Ad hoc Networks Using Artificial Intelligence”. Proc. HIS'03 conf. On Design and Application of Hybrid Intelligent Systems, pp.830-841, December 2003.

[8] C. E. Perkins and P. Bhagwat, "Highly Dynamic DestinationSequenced Distance-Vector Routing for Mobile Computers", ACM SIGCOMM, Oct. 1994, pp.234-244.

[9] M. Sheng, J. Li and Y. Shi, "Routing protocol with QoS guarantees for ad-hoc networks", Electronics Letters, Vol. 39 No.1, 9th January 2003.

[10] M.R. Pearlman and Z.J. Haas, "Determining the Optimal Configuration for the Zone Routing Protocol," IEEE Journal on Selected Areas in Communications, Special Issue on
Wireless Ad Hoc Networks, vol.17, no.8, pp.1395-1414, August 1999

[11] R.G. Gallager "A minimum delay routing algorithm using distributed computations", IEEE Transactions on Communications, Vol. COM-25, 1977.

[12] A.E. Ozdaglar, D. P. Bertsekas "Optimal Solution of Integer Multicommodity Flow Problem with Application in Optical Networks", Proc. Of Symposium on Global Optimisation, June 2003.

[13] E. Crawley, R. Nair, B. Rajagopalan, H. Sandick, " $A$ Framework for QoS-based Routing in the Internet", RFC2386, IETF, August 1998.

[14] E. Rosen, A. Viswanathan, R. Callon, "Multiprotocol Label Switching Architecture", Internet Draft draft-ietf-mpls-arch06.txt, IETF, August 1999.

[15] W. Stallings, "MPLS", Internet Protocol Journal, Vol. 4, nr. 3, September 2001.

[16] J. Strassner, "Policy-Based Network Management : Solutions for the Next Generation." Morgan-Kaufmann, 2003.

[17] M. Welzl, "Scalable Performance Signalling and Congestion Avoidance" Kluwer Academic Publishers, 2003.

[18] Z. Wang and J. Crowcroft, "QoS Routing for Supporting Resource Reservation". In IEEE Journal on Selected Areas in Communications, September 1996.

[19] R.S. Sutton and A. G. Barto, "Reinforcement Learning" MIT Press, 1997.

[20] S. Ziane and A. Mellouk "A Reinforcement Learning Approach for Routing and Scheduling Packets in Dynamic Networks". In proc of 1st IEEE International Conference on Information \& Communication Technologies: from Theory to Applications, April 2004.

[21] J. Boyan and M. L. Littman. "Packet routing in dynamically changing networks: a reinforcement learning approach". In Advances in Neural Information Processing Systems, volume 7, pages 671-678, 1994.

[22] S. Kumar and R. Miikkualainen, "Confidence-based Qrouting: an on-queue adaptive routing algorithm" In Proceedings of Neural Networks in Engineering, 1998.

[23] S. Kumar and R. Miikkualainen, "Dual reinforcement $Q$ routing: an on-queue adaptive routing algorithm" In Proceedings of Neural Networks in Engineering, 1997.

[24] P. Goetz, S. Kumar, R. Miikkulainen, “On-Line Adaptation of a Signal Predistorter through Dual Reinforcement Learning”. Proc. Machine Learning. Proceedings of the 13th Annual Conference Bari, Italy, 1996.

[25] E. Gelenbe, R. Lent, Z. Xu "Networking with Cognitive Packets", Proc. ICANN 2002, Madrid, Spain, August 27-30, 2002.

[26] D. Subramanian, P. Druschel, and J. Chen. "Ants and reinforcement learning: A case study in routing in dynamic networks", In Proceedings of the Fifteenth International Joint Conference on Artificial Intelligence, volume 2, pages 832-839, 1997. 
[27] S. Hoceini, A. Mellouk, Y. Amirat, "Neural Net Based Approach for Adaptive Routing Policy in Telecommunication Networks" In Lecture Notes in Computer Science, Springer-Verlag Heidelberg , ISBN: 0302-9743, Volume 3079, 2004.

[28] S. Hoceini, A. Mellouk, Y. Amirat, "K-Shortest Paths QRouting: A New QoS Routing Algorithm in Telecommunication Networks" In Lecture Notes in Computer Science, Springer-Verlag Heidelberg , ISBN: 0302-9743, Volume 3421, 2005.

[29] I. Kassabalidis, A.K. Das, M.A. El-Sharkawi, R.J. Marks II, P. Arabshahi, A. Gray, "Intelligent routing and bandwidth allocation in wireless networks", Proc. NASA Earth Science Technology Conf. College Park, MD, August 28-30, 2001.
[30] S.Ziane and A. Mellouk, "A Swarm Intelligent Scheme for Routing in Mobile Ad hoc Networks", Proc IEEE ICW 2005 on High Speed Networks, Montreal, Canada, August 2005.

[31] G. Di Caro and M. Dorigo, “ AntNet: distributed stigmergetic control for communication networks". Journal of Artificial Intelligence Research, vol.9, pp. 317-365, 1998.

[32] J.S.Baras and H. Mehta, " Dynamic Adaptive Routing in Manets" In Procedings of Annual ARL CTA Symposium, 2003.

[33] J.S. Baras and H. Mehta, "A Probabilistic Emergent Routing Algorithm (PERA) for Mobile Ad Hoc Networks", Proceedings of WiOpt '03: Modeling and Optimization in Mobile, AdHoc and Wireless Networks, Sophia-Antipolis, France, March 3-5, 2003. 